\title{
Epilogue
}

\section{The WHO multinational study of vascular disease in diabetes (WHO MSVDD)}

The papers in this Supplement to Diabetologia deal predominantly with the incidence of diabetes-related microvascular and macrovascular disease outcomes during the 7 to 9 years follow-up in 10 of the 14 centres of the original prevalence study [Diabetes Drafting Group (1985) Diabetologia 28: 615-640]. The content of each of the papers is summarised below.

E.T.Lee and H.Keen et al. describe the populations followed-up and they summarise the vascular disease outcomes. Of the 4729 subjects originally recruited at the 10 centres, $11.4 \%$ had died, $6.5 \%$ could not be relocated, $66.9 \%$ were seen and re-examined using the original ascertainment methodology and in $15.2 \%$ key outcome data was obtained from secondary sources. Marked differences between centres are shown for incident coronary heart disease and amputations and for risk of development of clinical proteinuria and renal failure, with Oklahoma American Indians at high risk for all. Retinopathy and visual impairment varied less. Subsequent papers deal with analysis of individual outcomes and their relation to baseline risk factors.

N.J.Morrish et al. compare mortality rates, causes of death and excess mortality expressed as standardised mortality ratios. There were major intercentre variations in mortality, resembling variation in the background non-diabetic populations. Cardiovascular disease accounted for $44 \%$ and $52 \%$ of deaths in Type I (insulin-dependent) diabetes mellitus, and Type II (non-insulin-dependent) diabetes mellitus, respectively and renal disease for $21 \%$ and $11 \%$. Age adjusted mortality rates and standardised mortality ratios were higher in Type I than Type II diabetes. Men and women from Tokyo had strikingly low excess mortality and all-cause mortality rates were particularly high in the Warsaw and Berlin centres.

H. Keen and E. T. Lee et al., using standardised direct ophthalmoscopy, present estimates of incidence of any retinopathy and proliferative retinopathy by centre and in pooled data in 2877 re-examined subjects who were free of them at baseline and relates their appearance to baseline 'risk factors'. Any retinopathy appeared in $47.7 \%$ and proliferative retinopathy in $9.7 \%$. In stepwise logisitc regression analyses, baseline systolic pressure and fasting plasma glucose were statistically significant risk factors for any retinopathy, and diabetes duration and fasting glucose for incident proliferative disease. Observer variation probably contributed to inter-centre incidence differences but not to important associations with baseline variables.

E. Miki et al. describe the incidence of severe visual impairment and prevalence at follow-up of all degrees of visual impairment using the same functional measures of vision employed at baseline. Crude overall incidence of severe visual impairment was $1.96 \%$; the variation between $0.30 \%$ and $3.45 \%$ in individual centres could have arisen by chance. Associated baseline variables included age at diagnosis, duration of diabetes, prior retinopathy, blood pressure, cholesterol and fasting glucose; the last four statistically significant in multivariate analyses. Prevalence of all grades of visual impairment at follow-up was $15.0 \%$. It differed significantly between centres and was variably associated with baseline retinopathy, renal disease, hypertension, diabetes duration and fasting glucose.

P.H.Bennett et al. report on increased urinary albumin (A) and creatinine (C) excretion in 8 of the 10 follow-up cohorts and measured centrally. Both 'microalbuminuria' (A/C ratio 30-299 mg/g) and 'macroalbuminuria' (A/C ratio $\geq 300 \mathrm{mg} / \mathrm{g}$ ) prevalences were highest in the 2 American Indian centres and lowest in London. Increased urinary albumin was significantly associated with systolic blood pressure in most centres, with insulin treatment and diabetes duration in some and with cigarette smoking in one. Increased urinary albumin was also significantly 
associated with coronary heart disease, stroke and lower extremity amputation in most centres and with retinopathy in five.

H.M. Colhoun et al. consider the incidence of renal failure in the 3558 subjects free of it at baseline. By the time of the follow-up examination, 53 of 959 Type I and 134 of 2599 Type II diabetic patients had entered renal failure, an overall incidence rate of 6.3 per 1000 patient years. Age and diabetes duration were associated with incident renal failure in Type I and Type II diabetes, age appearing more important in the latter. Systolic blood pressure was associated in Type I but not in Type II diabetic patients, but retinopathy and prior proteinuria was found strongly in both. Baseline fasting glucose and triglyceride concentrations were independently associated with incident renal failure in Type II diabetes but smoking showed no relation in this group.

J.H.Fuller et al. explore the baseline risk factors, classic and diabetes-related factors, for cardiovascular morbidity and mortality in the subjects originally recruited to the 10 follow-up centres. In both Type I and Type II diabetic patients, blood pressure, cholesterol and proteinuria significantly and independently predicted fatal and non-fatal myocardial infarction and stroke. In Type II diabetes, fasting triglyceride was independently related to cardiovascular disease in men was fasting glucose in both sexes. Retinopathy was associated with cardiovascular disease in Type I and Type II diabetes. Thus, both general population and diabetes-related factors were determinants of the risk of cardiovascular disease in this cohort.

N. Chaturvedi et al. deal with the incidence of lower limb amputation and/or gangrene, its risk factors and mortality associations in the 10 follow-up centres. Amputation rates varied enormously in Type I and Type II diabetes and were particularly high in the American Indian centres. Age, diabetes duration, sex and fasting glucose and triglyceride were signficantly associated baseline risk factors. Even after allowing for these, relative risk for American Indians compared with Europeans was over 10 for Type I and over 3 for Type II diabetes.

H. Keen and N. Morrish et al. assesse the contribution of observer variation in Minnesota coding of electrocardiograms to apparent deterioration and improvement in serial ECGs from the London cohort. Blind re-reading of a constructed sample of 118 paired baseline to follow-up ECG records showed little short-term (6 month) variation but some coding drift when the same records were re-read 11 years later, mainly involving some original over-reading of marginal baseline abnormalities. The results show that almost all of the reported ECG deterioration during follow-up and about half the apparent ECG improvement was confirmed.

E. T. Lee and M. Lu et al. compare the incidence of vascular complication in patients whose diabetes was diagnosed before age 30 in European, Asian and American Indian groups of the 10 follow-up centres. In these younger-diagnosed subjects, American Indians had higher rates of incident retinopathy, clinical proteinuria (and raised urinary albumin excretion), renal failure and lower extremity amputation than European and Asian patients. European women reported more incident angina than other cohorts.

The paper by Z.S. Chi et al. differs from the other Supplement papers in that it is a prevalence study of vascular complications in a cohort of Chinese people with diabetes from the Beijing area, assembled and examined precisely after the published WHO MSVDD protocol. Using the same methodology, compared with the 14 centres of the WHO MSVDD, the Chinese sample resembled the Hong Kong and Tokyo cohorts, differing dramatically from the others with considerably lower rates of prevalent cardiovascular disease, especially coronary heart disease but exhibiting significantly higher rates of retinopathy and nephropathy.

In conclusion the WHO Multinational Study of Vascular Disease in Diabetes established the feasibility of following an agreed common protocol to collect comparable data on the prevalence and incidence of diabetes associated complications from geographically, ethnically and culturally diverse diabetic populations. Limits are imposed on the interpretations and conclusions to be drawn from these studies by the specificity, sensitivity and accuracy of the simple, standardised instruments of ascertainment that were used, methods that have greatly advanced since this pioneering study was founded. Despite these limitations, however, major inter-centre differences in incidence and prevalence of the complications, particularly those of macrovascular origin, seem very likely. The emergence of statistically significant correlations between baseline characteristics and incident events, within centres and in pooled data, lends further credence to their reality. The nature and commonality of identifiable risk factors for the incidence of these vascular complications of diabetes in different patient groups supports the view that, to a great extent, they could be alleviated or prevented.

Harry Keen for the Multinational Group of Investigators 